\title{
A study on the clinical usefulness, validity, and test-retest reliability of the Spirokit, a device that combines the pulmonary function test and respiratory muscle strength test
}

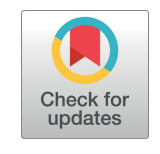

\author{
Byeong-Soo Kim ${ }^{a}$, , Myung-Mo Lee ${ }^{b(1)}$ \\ ${ }^{a}$ Department of Physical Therapy, Graduate School, Daejeon University, Daejeon, Republic of Korea \\ ${ }^{b}$ Department of Physical Therapy, Daejeon University, Daejeon, Republic of Korea
}

\begin{abstract}
Objective: This study was conducted to identify the clinical usefulness, validity, and reliability of the Spirokit, a device that com-
\end{abstract} bines the pulmonary function test (PFT) and respiratory muscle strength (RMS) test.

Design: Cross-sectional study.

Methods: Forty young adults (male: 23, female: 17) participated in a PFT and a RMS test. The concurrent validity for pulmonary function was assessed by comparing data obtained from MicroQuark and the Spirokit and the agreements between the MicroRPM and the Spirokit for RMS were compared. The test-retest reliability of the Spirokit was determined by comparing data obtained from the first and second sessions. The test and retest were performed at the same time after one day for the PFT and RMS test. Validity was estimated using intraclass correlation coefficients (ICCs), and by calculating $95 \%$ limits of agreement (LoA). To estimate interrater reliability, ICCs were calculated.

Results: The Spirokit showed a high agreement intra class coefficient (ICC [2, 1]): 0.978-0.999, 95\% limits of agreements (95\% LOA): -0.798 to 0.847 with MicroQuark. It also showed a high level of concordance ICC $(2,1): 0.992$ to $0.993,95 \%$ LOA: -9.313 to 11.169 with MicroRPM. The test-retest reliability of the Spirokit was analyzed using ICC $(2,1)$, and showed a high level of reliability (ICC $[2,1]=0.960$ to 0.998 ). Standard error of measurement \% (SEM\%) was $0.12 \%$ to $3.39 \%$, and minimum detectable change $\%$ (MDC\%) was $0.02 \%$ to $3.79 \%$, indicating high level of reliability.

Conclusions: The Spirokit is a device with high validity and reliability that can be used to simultaneously measure PFT and RMS tests.

Key Words: Equipment and supplies, Lung diseases, Pulmonary function test, Reproducibility of results, Respiratory muscles

\section{Introduction}

The Republic of Korea has the highest concentration of air pollution and fine dust among Organization for Economic Cooperation and Development (OECD) countries due to rapid industrialization. The resulting number of premature deaths is projected to be highest among OECD countries, from 359 per million in 2010 to 1,109 per million in 2060 [1]. Chronic obstructive pulmonary disease (COPD) refers to a condition in which respiratory tract abnormalities such as coughing, sputum, and difficulty breathing are irreversible as the airways become narrowed or closed. It is the third leading cause of death worldwide due to its high prevalence rate [2]. The domestic mortality rate of this disease is ranked seventh among all diseases, and it is known that the prevalence rate of the disease is $13.6 \%$, which is higher than diabetes or stroke. The prevalence of COPD is expected to gradually increase due to air pollution [3]. The resulting socio-economic costs are also estimated to be five to ten times higher than that of diabetes and hypertension [4].

Received: 28 May, 2020 Revised: 18 June, 2020 Accepted: 19 June, 2020

Corresponding author: Myung-Mo Lee (ORCID https://orcid.org/0000-0002-2192-1701)

Department of Physical Therapy, Daejeon University, 62 Daehak-ro, Dong-gu, Daejeon 34520, Republic of Korea

Tel: 82-42-280-4295 Fax: 82-42-280-2295 E-mail: mmlee@dju.kr

(c) This is an Open-Access article distributed under the terms of the Creative Commons Attribution Non-Commercial License (http://creativecommons.org/licenses/ by-nc/4.0) which permits unrestricted non-commercial use, distribution, and reproduction in any medium, provided the original work is properly cited.

Copyright @ 2020 Korean Academy of Physical Therapy Rehabilitation Science 
Asthma is a major respiratory disease in children and adolescents [5]. Children and adolescents undergo rapid changes during their growth and development, leaving them with undeveloped bodies, tissues, and weak immune systems. This makes them more susceptible to fine dust because they have more air intake when it comes to their body size than adults [6]. Children in the Republic of Korea have a prevalence rate of $10.5 \%$ for asthma, while adolescents have a prevalence rate of $7.5 \%$. Therefore, the survey showed that asthma had the highest disease burden rate in children and adolescents [3].

Chronic respiratory disease is not easy to detect due to the weak initial symptoms and the nature of the disease. It is usually discovered when the main symptoms appear and treatment is given. However, there is a greater difference in disease progression and prognosis than in the early detection. Although chronic respiratory disease requires constant management in daily life, the examination equipment and management system are mostly concentrated in hospitals and are therefore not managed by the community health system [7].

Pulmonary function tests (PFTs) are used to objectively measure an individual's ability to breathe and also measures how well the lungs ventilate and exchange gas. These tests are essential for the diagnosis of respiratory diseases [8] The main data calculated from the PFT includes the following values: static vital capacity (SVC), forced vital capacity (FVC), forced expiratory volume (FEV), forced expiratory volume in one second $\left(\mathrm{FEV}_{1}\right), \%$ of forced expiratory volume in one second $\left(\mathrm{FEV}_{1} \%\right)$, forced expiratory flow between $25 \%$ and $75 \%\left(\mathrm{FEF}_{25-75 \%}\right)$, maximal voluntary ventilation (MVV), and peak expiratory flow (PEF). FVC, $\mathrm{FEV}_{1}$, and $\mathrm{FEV}_{1} \%$ values are the most important indicators for screening for respiratory diseases and understanding their functions [9]. The PEF value is the main criterion for determining the level of sputum discharge ability and expiration function [10].

The respiratory muscle strength (RMS) test is a non-invasive and easy tool used to assess the function of the muscles involved in breathing. This is a test that indirectly measures the respiratory muscle through the inhalation and exhalation pressure values. The RMS test is important for evaluating ventilation capacity and respiratory muscle weakness [11]. The data of the RMS test is composed of the maximum inspiratory pressure (MIP) and the maximum expiratory pressure (MEP). MIP is used as an indicator to measure the strength of the muscles related to inhalation such as the diaphragm, while the MEP is used as an indicator to evaluate the strength of the muscles related to expiration, such as the intercostal and abdominal muscles [12].

Currently used PFTs and RMS tests are mainly conducted in large hospitals or specialized hospitals for respiratory diseases. These tests are generally not included in general health examinations conducted by the National Health Insurance Corporation, which has led to a decrease in the rate of chronic respiratory disease screening [3]. In particular, the measurement of the respiratory muscle affects the diagnosis of lung capacity and chronic respiratory disease. However, it is not a very well-known test, and it is difficult to measure as different devices and components must be equipped separately [13].

Patients with other chronic diseases have several personal care products such as blood glucose meters and blood pressure monitors. However, only an analog-type peak flow meter for measuring PEF is used as a personal device for those suffering from chronic respiratory disease [14]. The lack of devices makes it difficult for users as they have to write the records manually. There are also some shortcomings with only using the PEF value to examine and manage respiratory health conditions [15].

With the recent development of information technology, various treatment and inspection devices are being developed in the medical field. In particular, various medical devices are being developed in the form of a software that is applicable to smartphones and notebooks that have become popular among the public [16]. The development of medical devices converged with IT technology not only increases the convenience for users, but also the convenience for examiners, contributing to the management and treatment of diseases [17]. Respiratory health-related items are currently being developed as portable respiratory trainers that work with smartphones and personal PCs [16]. However, most portable respiratory trainers that need to be available for practical use are difficult to find in Korea. The reason for this is mainly because these devices exist as training devices and do not include ways to check results. Even though portable respiratory training devices are being developed overseas, they are difficult for the general public to obtain due to the high cost of imported products. In addition, despite the fact that Korean pulmonary function predictions have been developed, the PFT device used in clinical practice is not suitable for Koreans because it uses Morris' Asian formula [18].

Therefore, the goal of this study was to prove the usefulness of the Spirokit device, a PFT device developed to measure PFTs and RMS tests using sensors and IT technology. The study compared and analyzed the validity be- 
tween the devices and the test-retest reliability by using the PFT and RMS test devices currently used in clinical practice.

\section{Methods}

\section{Participants}

Forty-one subjects in their twenties from Daejeon were selected to participate in the study. The selection criteria was those who had no history of chronic respiratory diseases such as asthma, chronic bronchitis, and lung emphysema, those who had no history of surgical operation on the chest wall, or those who had no previous PFTs. Those who were currently diagnosed and being treated for respiratory diseases such as colds, and those who complained of dizziness and chest pain during the examination were excluded. The purpose and procedure of the study were explained to all participants. The study was conducted on those who understood the information and voluntarily agreed to participate in the study. The study was approved by the Daejeon University Institutional Review Board (1040647-202004-HR003-03).

\section{Procedures and instruments}

To verify the validity of the measurement equipment, pulmonary function and respiratory muscle were measured using a standard PFT device, the MicroQuark (Cosmed, Rome, Italy) and a respiratory muscle measurement device, the MicroRPM (Carefusion, San Diego, CA, USA). After a five minute pause after the measurement, the PFT and RMS test were performed using the Spirokit to compare the measured valuess.

The MicroQuark is a device that was developed according to the PFT device guidelines suggested by the American Thoracic Society (ATS) [9]. It is recognized as the gold standard device for PFT devices. It can detect volumes up to $12 \mathrm{~L}$ and has a flow rate range of 0.03-20 L/s. It has an accuracy of an error range of $\pm 3 \%$ when measuring $50 \mathrm{~mL}$. The size of the device is $45 \mathrm{~mm}$ in length, $150 \mathrm{~mm}$ in height, and $53 \mathrm{~mm}$ in width, with a weight of $75 \mathrm{~g}$. The device is connected to a PC through a USB port where results can be uploaded and measured through a OMNIA software Ver. 1.1 (Cosmed, Roma, Italy). The software measures SVC, FVC, $\mathrm{FEV}, \mathrm{FEV}_{1}, \mathrm{FEV}_{1 \%}, \mathrm{FEF}_{25-75 \%}$, PEF, and MVV. Only FVC, $\mathrm{FEV}_{1}$, and PEF values among the measurement indicators were recorded as data for the study.

Before the test, calibration was performed with a $3 \mathrm{~L}$ syringe [19]. The measurement method required participants to inhale as much as possible and then exhale for six seconds under maximum effort [20].

The MicroRPM (Figure 1) is a respiratory test device that has been verified for precision. IT is used to indicate the muscular strength of muscles used in the respiratory system as pressure values. The size of the device is length $60 \mathrm{~mm} \times$ height $170 \mathrm{~mm} \times$ width $26 \mathrm{~mm}$, and it weighs $175 \mathrm{~g}$. It has a measurement pressure range of $\pm 300 \mathrm{cmH}_{2} \mathrm{O}$, and an accuracy of $\pm 3 \%$ for its error range [21]. This device intuitively displays a specific value, but the instrument must be reset and the intermediate filter must be replaced each time the inlet/outlet of air pressure is measured. While the intraoral pressure was excluded as much as possible, the respiratory muscle was measured by using only the muscles around the diaphragm, chest wall, and neck during MIP measurement. When measuring MEP, only the muscles around the abdomen and chest wall were used for maximum spitting [22].

The Spirokit (TR Ltd., Daejeon, Korea; 2020) is a self-

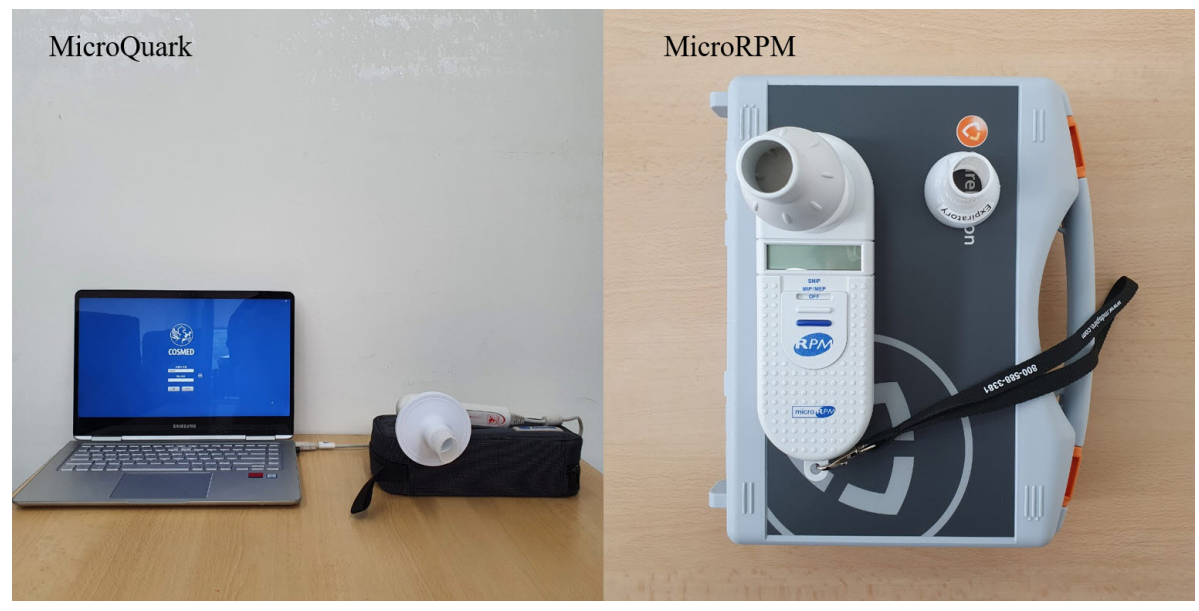

Figure 1. MicroQuark and MicroRPM. 
developed wireless PFT device that enables pulmonary vitality testing and respiratory muscle measurement using hall sensors and pressure sensors (Figure 2). The device uses a method to measure the propeller rotation speed by applying a magnet to the propeller after energizing the hall sensor. It can also be used to measure the speed and amount of gas inflow and outflow in each direction. For this study, the Spirokit was manufactured using a hall sensor able to measure a flow rate of up to $10 \mathrm{~L}$. The device has been manufactured to measure RMS using a pressure sensor when the back part of the hall sensor located on the head of the device was blocked with a stopper. The pressure sensor can measure $\pm 350 \mathrm{hPa}$ and has an error range of $\pm 1 \mathrm{hPa}$. In addition, it is possible to set the zero point automatically by measuring the atmospheric pressure in real time. $\mathrm{A} 1 \mathrm{hPa}$ is converted to $1.019763 \mathrm{cmH}_{2} \mathrm{O}$, and the number below the decimal point is rounded up from the first digit to change the unit to $\mathrm{cmH}_{2} \mathrm{O}$.

A printed circuit board was produced so that the data collected through the two sensors could be processed in a microcontroller unit and transmitted wirelessly through a Bluetooth sensor. This device is designed to be able to calculate the FVC, $\mathrm{FEV}_{1}$, and PEF values, which are the main indicators of the PFTs. It was also designed to measure MIP and MEP, the RMS test indicators. The measured data is relayed to a smartphone or laptop screen via Bluetooth. The results of the experiment were relayed through the Bluetooth
Chat App of the Samsung Galaxy S10 smartphone. Before the test, a syringe was used to inject $3 \mathrm{~L}$ of air for calibration. Abnormal data was corrected using the open source software Arduino IDE Sketch (Arduino, Strambino, Italy). The experiment was then conducted on the participants (Figure $1)$.

To measure the test-to-retest reliability of the Spirokit, a PFT and a RMS test were performed using the device at the same time the day after the test was conducted. Each measurement value was then compared. All tests were conducted after one instance of pre-training and rehearsal. The maximum value was recorded by measuring each value three times with each measuring device.

All tests were performed according to the guidelines for PFTs [9] presented by the ATS. Participants were seated in chairs without armrests or wheels and were instructed to look forward. Participants were instructed to keep their feet on the ground and maintain a 90-degree angle with their knee and hip joints as much as possible. Nose clips were used to prevent air from leaking through the nose. Participants were given a mouthpiece to bite down on, and their mouths were examined to see if any air was coming through the lips. If a participant felt dizzy or tired during the test, he/she was given a break, and tests were immediately halted for participants that wanted to stop. Data was collected on the final 40 participants, while the data for one of the original 41 participants was excluded due to withdrawing from the test.

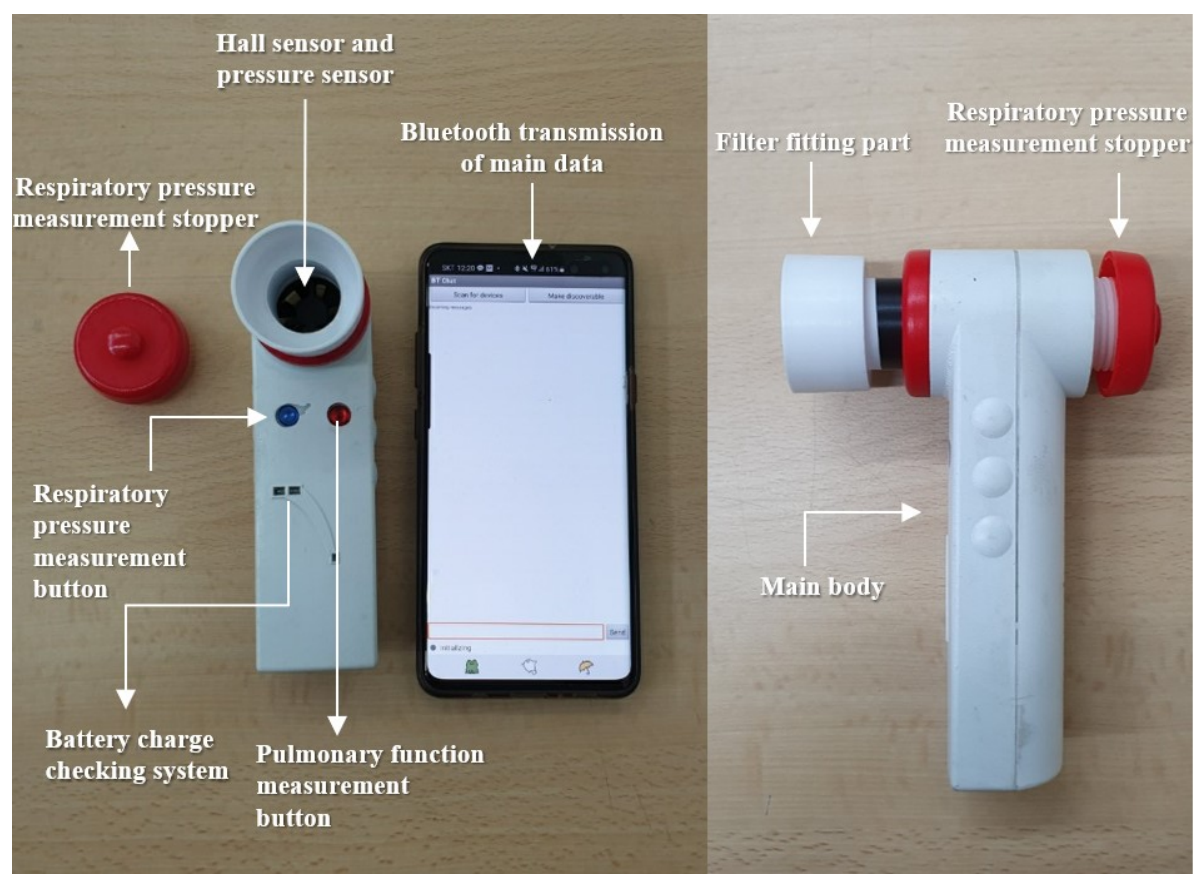

Figure 2. The Spirokit. 


\section{Statistical analysis}

The result value between each measuring device was descripted by the mean and standard deviation (SD). The differences between the measuring devices were statistically analyzed by the paired t-test.

Intra class coefficient (ICC $[2,1]$ ) was used to calculate data collected from MicroQuark, MicroRPM, and the concurrent validity of each variable of the Spirokit. If the result of the ICC value was less than 0.750 , it was said to be medium or low (moderate). It was said to be good for 0.750 to 0.900 , and excellent for more than 0.900 [23].

To thoroughly compare the measured values between devices, coefficients of variation of method errors $\left(\mathrm{CV}_{\mathrm{ME}}\right)$ and 95\% limits of agreements (95\% LOA) were calculated [24]. The measured values between devices were then displayed in diagram form using the Bland Altman Graph. $\mathrm{CV}_{\mathrm{ME}}$ data calculated the coefficient of variation using SD calculated from the data of each device and converted it into a percentage $\left(\mathrm{ME}=\mathrm{Sd} / \sqrt{2}, \mathrm{CV}_{\mathrm{ME}}=2 \mathrm{ME} /(\mathrm{X} 1+\mathrm{X} 2) \times 100 \%\right)$.

Paired t-tests were performed to determine the statistical difference between the Spirokit's measurement values between test and retest. $\operatorname{ICC}(2,1)$ was used for the test-retest reliability analysis. The standard error of measurement (SEM) was calculated as $\mathrm{SD} \times \sqrt{(1-\mathrm{ICC})}$ using the higher of the two SD values. For convenience of data interpretation, SEM was expressed as the average SEM percentage (SEM\%) [25].

In addition, the minimum detectable change (MDC) was

Table 1. General characteristics of participants $\quad(\mathrm{N}=40)$

\begin{tabular}{lc}
\hline \multicolumn{1}{c}{ Variable } & Value \\
\hline Sex $($ male/female $)$ & $23 / 17$ \\
Age $(\mathrm{y})$ & $22.30(2.01)$ \\
Height $(\mathrm{cm})$ & $168.32(9.40)$ \\
Weight $(\mathrm{kg})$ & $66.38(14.11)$ \\
BMI $\left(\mathrm{kg} / \mathrm{m}^{2}\right)$ & $23.18(3.24)$ \\
\hline
\end{tabular}

Values are presented as number only or mean (SD). BMI: body mass index. calculated by calculating $1.96 \times \mathrm{SEM} \times \sqrt{2}$ to confirm that the participant's measurement data appeared at a confidence level of $95 \%$. The calculated MDC was then converted to a percentage of the mean before MDC 95\% was calculated [25].

Data were analyzed using Microsoft Excel 2018 (Microsoft, Redmond, WA, USA). The 95\% LOA was derived using Medicalc ver. 19.3.1 (Medicalc software, Ostend, Belgium). The statistical significance analysis of the measured values, the statistical analysis of the concordance degree between devices, and the reliability between test and retest were all analyzed using IBM SPSS Statistics for Windows, Version 25.0 (IBM Co., Armonk, NY, USA). All statistical significance levels $(\alpha)$ were set at 0.05 .

\section{Results}

The general characteristics of the participants were as follows: participants were made up of 23 males and 17 females, the average age was 22.30 years, the average height was $168.32 \mathrm{~cm}$, the average weight was $66.38 \mathrm{~kg}$, and the average body mass index was $23.18 \mathrm{~kg} / \mathrm{m}^{2}$ (Table 1 ).

There was no significant difference between the measurement results of the PFT measured through the MicroQuark and Spirokit devices and the paired t-test $(p>0.05)$. The correlation coefficient between the two devices was $0.999(0.999-$ 1.000) for ICC $(2,1)$ of FVC, 0.997 (0.994-0.998) for ICC $(2,1)$ of $F_{1}$, and $0.988(0.978-0.994)$ for $\operatorname{ICC}(2,1)$ of PEF, which showed a high concordance degree. The $\mathrm{CV}_{\mathrm{ME}} \%$ value of each variable was derived as small as FVC 0.01, $\mathrm{FEV}_{1}$ 0.02 , and PEF $0.03 \%$. Each 95\% LOA was FVC - 0.114 to $0.079, \mathrm{FEV}_{1}-0.162$ to 0.156 , and PEF -0.798 to 0.847 , which showed that the results were mostly symmetrical (Table 2, Figure 3).

The results of the respiratory muscle measurements taken by the MicroRPM and the Spirokit device were analyzed by the paired t-test $(p>0.05)$. There was no significant difference between the measurement results. The concordance de-

Table 2. Level of agreement of the pulmonary function test variables for participants using the MicroQuark and the Spirokit

\begin{tabular}{lccccc}
\hline \multicolumn{1}{c}{ Variable } & MicroQuark & Spirokit & ICC $[2,1](95 \%$ CI $)$ & CV $_{\text {ME} \%}$ & $95 \%$ LOA \\
\hline FVC $(\mathrm{L})$ & $4.24(0.96)$ & $4.25(0.96)$ & $0.999(0.999-1.000)$ & 0.01 & $-0.114-0.079$ \\
FEV $_{1}(\mathrm{~L})$ & $3.60(0.71)$ & $3.60(0.71)$ & $0.997(0.994-0.998)$ & 0.02 & $-0.162-0.156$ \\
PEF (L/s) & $8.60(1.88)$ & $8.59(1.98)$ & $0.988(0.978-0.994)$ & 0.03 & $-0.798-0.847$ \\
\hline
\end{tabular}

Values are presented as mean (SD).

ICC: intra correlation coefficient, CI: confidence interval, $\mathrm{CV}_{\mathrm{ME}} \%$ : coefficients of variation of method error \%, 95\% LOA: $95 \%$ limits of agreements, FVC: forced vital capacity, $\mathrm{FEV}_{1}$ : forced expiratory volume in 1 second, PEF: peak expiratory flow. 

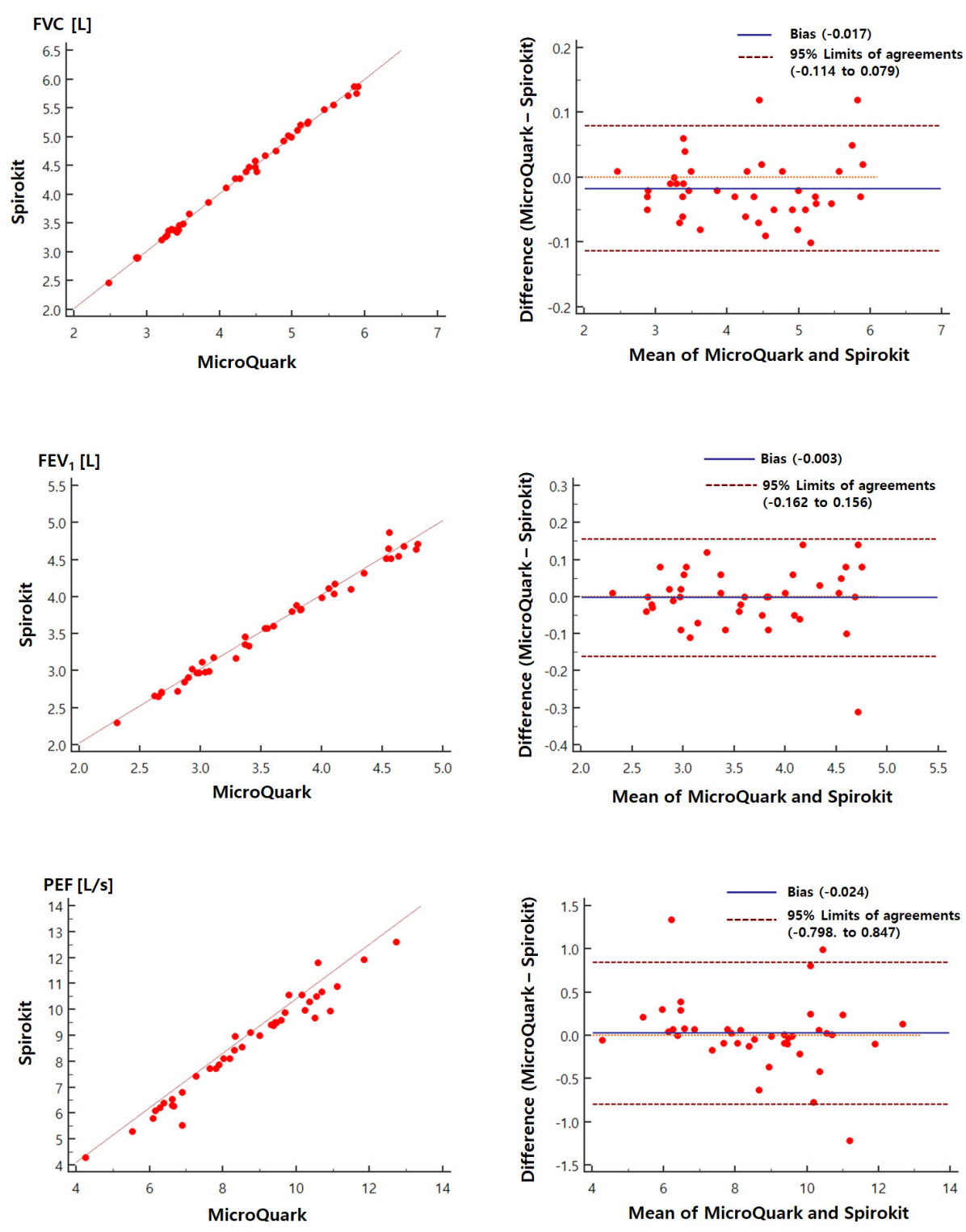

Figure 3. Relationship between Micro Quark and the Spirokit for pulmonary function test variables in participants. FVC: forced vital capacity, PEF: peak expiratory flow, $\mathrm{FEV}_{1}$ : forced expiratory volume in 1 second.

Table 3. Level of agreement of the respiratory muscle strength test variables for participants using the MicroRPM and the Spirokit

\begin{tabular}{cccccc}
\hline Variable & MicroRPM & Spirokit & ICC $[2,1](95 \%$ CI $)$ & CV $_{\text {ME } \%}$ & $95 \%$ LOA \\
\hline $\mathrm{MIP}\left(\mathrm{cmH}_{2} \mathrm{O}\right)$ & $91.35(29.48)$ & $90.38(28.69)$ & $0.992(0.985-0.996)$ & 0.04 & $-9.219-11.169$ \\
$\mathrm{MEP}\left(\mathrm{cmH}_{2} \mathrm{O}\right)$ & $74.55(20.81)$ & $75.95(21.71)$ & $0.993(0.987-0.996)$ & 0.04 & $-9.313-6.513$ \\
\hline
\end{tabular}

Values are presented as mean (SD).

ICC: intra correlation coefficient, $\mathrm{CI}$ : confidence interval, $\mathrm{CV}_{\mathrm{ME}} \%$ : coefficients of variation of method error \%, 95\% LOA: 95\% limits of agreement, MIP: maximal inspiratory pressure, MEP: maximal expiratory pressure.

gree between the two devices showed a high concordance rate of $0.992(0.985-0.996)$ for the MIP's ICC $(2,1)$, and $0.993(0.987-0.996)$ for the MEP's ICC $(2,1)$. The $\mathrm{CV}_{\mathrm{ME}} \%$ of each variable was derived as small as $0.04 \%$ for both MIP and MEP. Each 95\% LOA was MIP -9.219 to 11.169, MEP -9.313 to 6.513 , showing that the results were mostly sym- metrical (Table 3, Figure 4).

The test-retest measurement results measured through the Spirokit were analyzed through the paired t-test. As a result, there was no significant difference in the measurement results $(p>0.05)$. The correlation coefficient during the test-retest was $0.998(0.996-0.999)$ for ICC $(2,1)$ of FVC, the ICC 

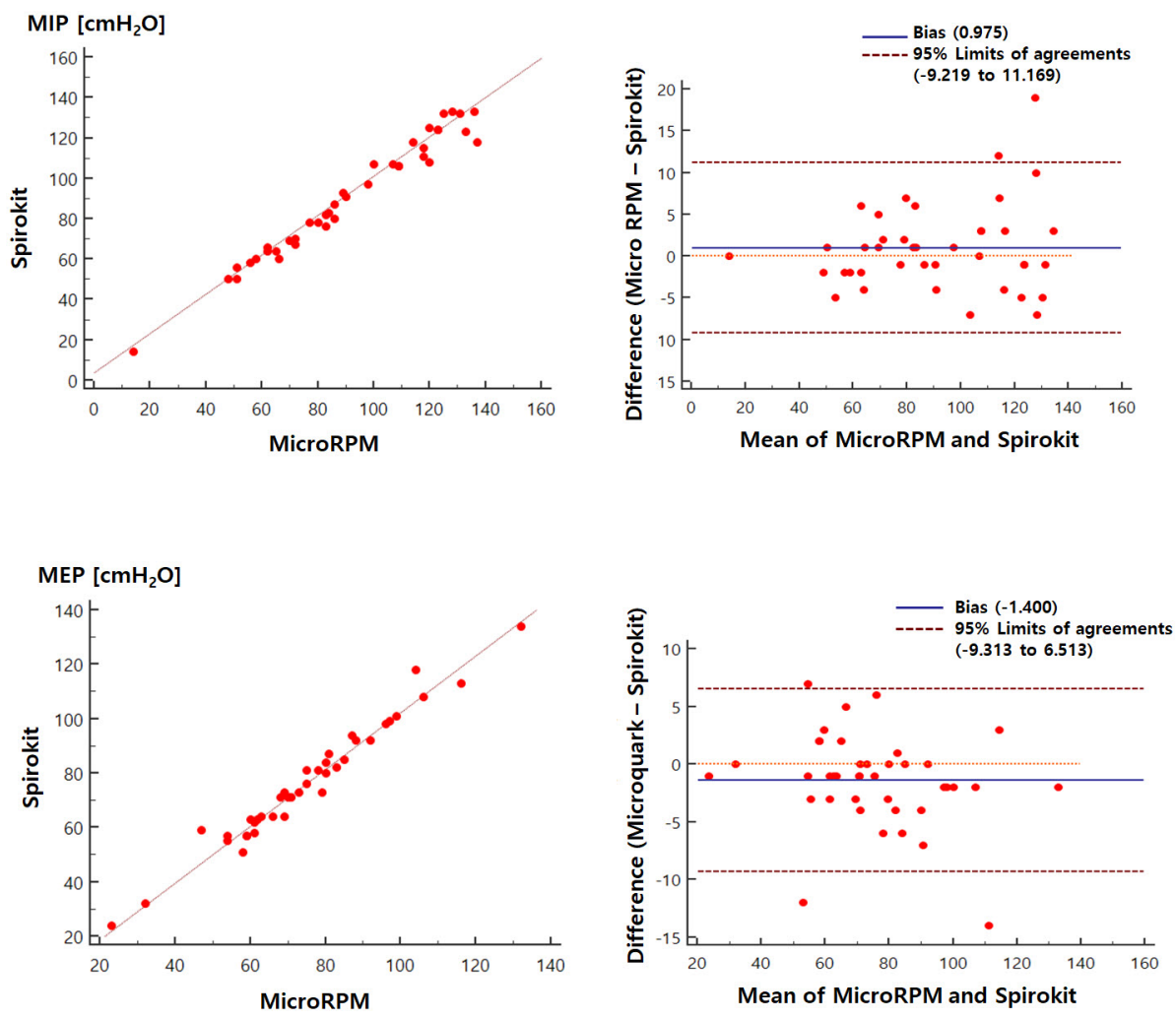

Figure 4. Relationship between MicroRPM and the Spirokit for respiratory muscle strength test variables in participants. MIP: maximal inspiratory pressure, MEP: maximal expiratory pressure.

$(2,1)$ of $\mathrm{FEV}_{1}$ was 0.993 (0.986-0.996), and the ICC of PEF $(2,1)$ was 0.960 (0.925-0.979), which showed a high concordance rate. For each $95 \%$ LOA, FVC -0.114 to 0.079 , $\mathrm{FEV}_{1}-0.162$ to 0.156 , and PEF -0.798 to 0.847 , showing that the results were mostly symmetrical (Figure 5). The $\mathrm{CV}_{\mathrm{ME}} \%$ of each variable was derived to be a small value of $0.02 \%, 0.02 \%$, and $0.06 \%$ of $\mathrm{FVC}, \mathrm{FEV}_{1}$, and PEF, respectively. For the SEM\%, FVC $0.12 \%, \mathrm{FEV}_{1} 0.53 \%$, and $\mathrm{PEF} 3.39 \%$ were found. The MDC $\%$ was $\mathrm{FVC} 0.02 \%, \mathrm{FEV}_{1}$ $0.03 \%$, and PEF $0.42 \%$, indicating that there was a strong and absolute test-retest reliability. The $\operatorname{ICC}(2,1)$ of MIP was 0.975 (0.953-0.987), and the ICC $(2,1)$ of MEP was 0.968 (0.939-0.983), showing a high reliability value. In addition, each 95\% LOA was mostly symmetrical with MIP - 18.40315.905 and MEP - 16.527-13.577 (Figure 6). The $\mathrm{CV}_{\mathrm{ME}} \%$ of each variable was derived as a small value of $0.07 \%$ of MIP and $0.07 \%$ of MEP. For the SEM $\%$, the MIP was $1.09 \%$, and MEP was $0.92 \%$. The MDC\% were MIP $3.79 \%$ and MEP 3.76\% (Table 4).

\section{Discussion}

Indicators of PFTs and RMS tests play an important role in determining respiratory health status. Early diagnosis is important as it can help identify chronic respiratory diseases with weak initial symptoms [9]. It is necessary to frequently check the respiratory health of those suffering from chronic respiratory diseases. However, there is a problem in that it is necessary to visit a specialized medical institution equipped with a device in order to receive a PFT and a RMS test. As the PFT device and RMS test device are provided separately, it is more difficult to perform the RMS test as it is not covered by health insurance. The Spirokit used in this study was developed through IT technology using sensors to solve usability and accessibility problems of existing PFT devices and RMS test devices.

This study aimed to investigate the concurrent validity and test-retest reliability of the Spirokit device when the sensor was developed in combination with 40 adults in their twenties. MicroQuark, a PFT device, and MicroRPM, a RMS test device, were used in clinical trials to gather statistics. A comparative analysis was conducted to investigate the concurrent validity and test-retest reliability.

In the study results, the FVC, $\mathrm{FEV}_{1}$, and PEF, all PFT values of the Spirokit, showed a very high concordance rate of $0.999,0.997$, and 0.988 , respectively. The $95 \%$ LOA is a reference data for determining the symmetry of the result value by displaying the variables as dots. As a result of this study, 

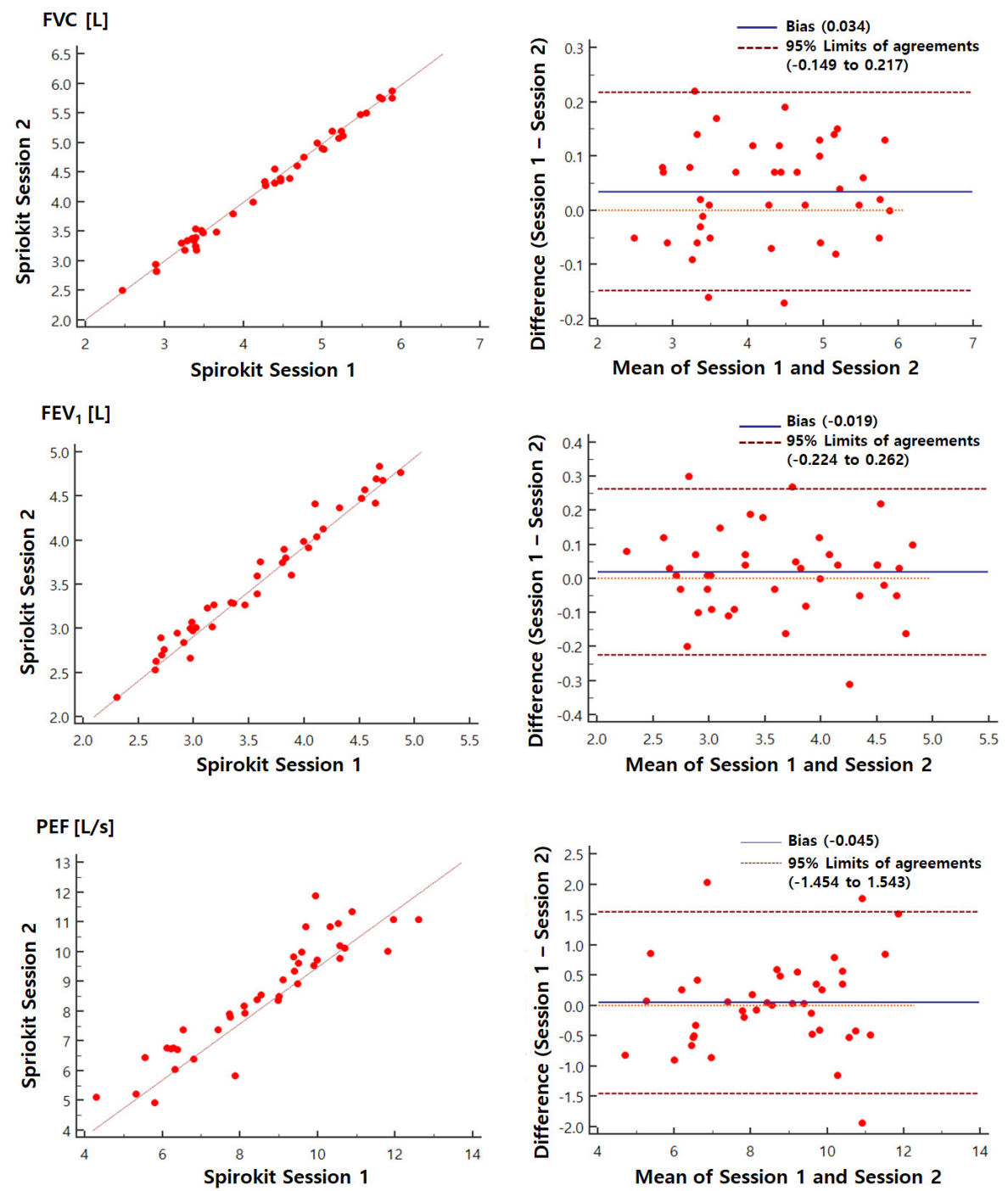

Figure 5. Agreement for the pulmonary function test variables between session 1 and session 2 of the Spirokit in participants. FVC: forced vital capacity, $\mathrm{FEV}_{1}$ : forced expiratory volume in 1 second, PEF: peak expiratory flow.

the values for each variable were FVC -0.114 to 0.079 , $\mathrm{FEV}_{1}-0.162$ to 0.156 , and $\mathrm{PEF}-0.798$ to 0.847 . Most of the results showed symmetry [24]. $\mathrm{CV}_{\mathrm{ME}} \%$ is data that interprets the degree of variation between scores that cannot be shown in correlation [23]. In the results of the PFT, $\mathrm{CV}_{\mathrm{ME} \%}$ showed a small variability with $0.01 \%, 0.02 \%$, and $0.03 \%$ for each variable. It uses the hall sensor with the current and electrodes to determine the propeller speed and calculates the air volume and amount of propeller rotations according to the speed. This is thought to be because it was developed with a principle similar to MicroQuark's principle of calculating the flow rate and velocity of air flowing through a propeller [26].

In addition, in the Spirokit, MIP and MEP, respiratory strength test values, MicroRPM, the existing respiratory strength test device, and the concordance rate for each varia- ble showed high concordance rates of 0.992 and 0.993 . Even at $95 \% \mathrm{LOA}$, the results were symmetrical with -9.219 to $11.169,-9.313$ to 6.513 for each variable. In addition, $\mathrm{CV}_{\mathrm{ME}} \%$ showed a small variability at $0.04 \%$ level in both MIP and MEP. This was composed of a similar principle that the pressure sensor of both the Spirokit and MicroRPM performed zero adjustments according to air pressure. The pressure value was then calculated through automatic real-time measurement of the internal sensor circuit according to the intake and exhalation pressure. This small variability was considered to be a result [27]. When the Spirokit was manufactured, it was used to find the principle of various test devices, measure air flow rate and speed, and measure real-time pressure to find and combine sensors capable of zero adjustment. After that, it was considered that the data value measured by the CPU was produced in a way that could be immediately 

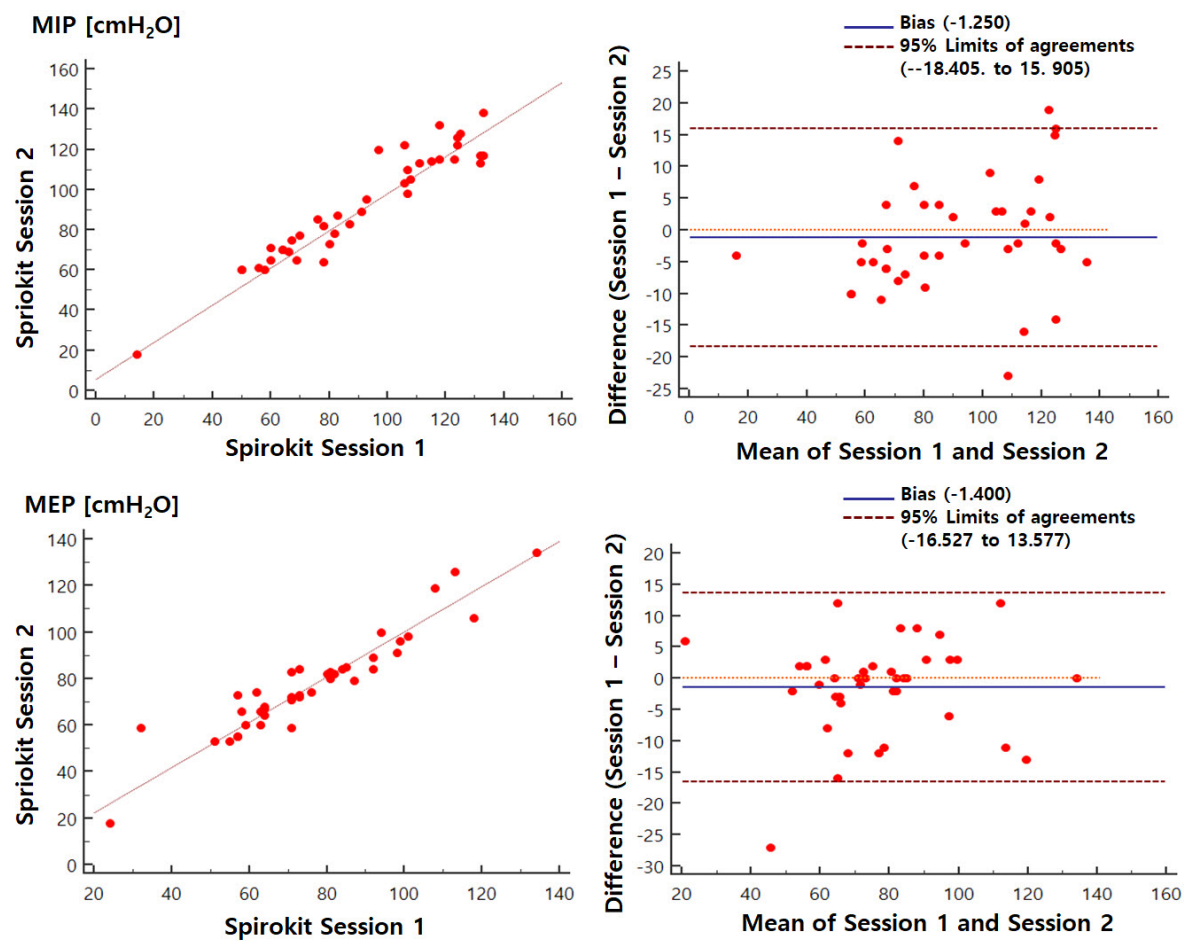

Figure 6. Agreement for the respiratory muscle strength test variables between session 1 and session 2 of the Spirokit in participants. MIP: maximal inspiratory pressure, MEP: maximal expiratory pressure.

Table 4. Test-retest reliability of the Spirokit variables for the two measurement sessions in participants

$(\mathrm{N}=40)$

\begin{tabular}{lccccccc}
\hline \multicolumn{1}{c}{ Variable } & Session 1 & Session 2 & ICC $[2,1](95 \% \mathrm{CI})$ & 95\% LOA & CV $_{\text {ME} \%}$ & SEM\% & MDC\% \\
\hline FVC (L) & $4.25(0.96)$ & $4.22(0.95)$ & $0.998(0.996-0.999)$ & $-0.149-0.217$ & 0.02 & 0.12 & 0.01 \\
FEV1 (L) & $3.60(0.71)$ & $3.58(0.72)$ & $0.993(0.986-0.996)$ & $-0.224-0.262$ & 0.02 & 0.53 & 0.03 \\
PEF (L/s) & $8.59(1.98)$ & $8.54(1.88)$ & $0.960(0.925-0.979)$ & $-1.454-1.543$ & 0.06 & 3.39 & 0.42 \\
MIP $\left(\mathrm{cmH}_{2} \mathrm{O}\right)$ & $90.38(28.69)$ & $91.63(26.51)$ & $0.975(0.953-0.987)$ & $-18.403-15.905$ & 0.07 & 1.09 & 3.79 \\
MEP $\left(\mathrm{cmH}_{2} \mathrm{O}\right)$ & $75.95(21.71)$ & $77.43(21.11)$ & $0.968(0.939-0.983)$ & $-16.527-13.577$ & 0.07 & 0.92 & 3.76 \\
\hline
\end{tabular}

Values are presented as mean (SD).

ICC: intra correlation coefficient, CI: confidence interval, 95\% LOA: $95 \%$ limits of agreements, $\mathrm{CV}_{\mathrm{ME}} \%$ : coefficients of variation of method error \%, SEM\%: standard error of measurement \%, MDC\%: minimum detectable change $\%$, FVC: forced vital capacity, $\mathrm{FEV}_{1}$ : forced expiratory volume in 1 second, PEF: peak expiratory flow, MIP: maximal inspiratory pressure, MEP: maximal expiratory pressure.

displayed, resulting in a high matching rate between devices.

The Spirokit's test-retest reliability analysis showed high reliability (ICCs $>0.968$ ) for all variables. $\mathrm{CV}_{\mathrm{ME}} \%$ for all variables was $0.02 \%$ to $0.07 \%$, and $95 \%$ LOA values were symmetrically distributed. Most of the variables were distributed within a narrow range. The research results were calculated through SEM and MDC 95\% to indicate the reliability between test-retest. SEM is a standard error estimation method between measurements in which the same participant occurs repeatedly by a specific test [25]. In the results of this study, it was expressed as a percentage of the average value (SEM\%). It was calculated at a range of $0.12 \%$ to $0.42 \%$ in all variables, and the measurement error range was low. MDC $95 \%$ is the minimum amount of change in score that can be regarded as a change in an individual rather than an error in measurement in a measurement tool. It was used to observe whether the participant's measurement environment and physical conditions changed between the primary and secondary tests.

In this study, MDC 95\% was also found to be small in the range of $0.01 \%$ to $3.79 \%$ in all variables. This showed that there were only small changes in the participant's test environment and physical conditions between the primary and secondary tests. Based on the results mentioned above, it was possible to explain the high agreement rate between test-retest in the study. These results were conducted at the same time for examination and retest to control the variables 
of lung function and RMS of the study participants. These were regarded as the result of keeping the measurement posture of each participant as high as the ATS standard [9]. The inspection environment was also considered to be the result of not providing as many spatial variables as possible for the primary and secondary tests by proceeding with the tests at the same space and temperature.

However, there were some differences in pulmonary function values when comparing the results to the average values of the RMS test. The filter used in the PFT conducted by the Spirokit and MicroQuark uses the same type of oval mouthpiece filter with a width of $30 \mathrm{~mm}$ and a diameter of $20 \mathrm{~mm}$. However, in the case of MicroRPM, it was considered that the participant felt a sense of heterogeneity with respect to the mouth filter during the examination by using a circular mouthpiece filter with a width of $20 \mathrm{~mm}$ and a diameter of $20 \mathrm{~mm}$.

The limitation of this study was that it was difficult to explain the conclusions based on the results of the study to various age groups as the tests were only conducted on healthy participants in their twenties. In addition, there were limitations in that it was difficult to generalize the results of this study to patients with respiratory diseases because studies have not been conducted on patients with respiratory diseases. Therefore, this study was somewhat limited to explain the reliability and validity of this device in patients with respiratory diseases.

In the future, further studies should be conducted to supplement the limitations to prove the validity and reliability of the Spirokit in more comprehensive participants.

In conclusion, the study was conducted to prove the validity and reliability of the Spirokit, a simple test device capable of performing the functions of the PFT and the RMS test. The results of the study conducted on men and women in their twenties showed that the Spirokit was a device with high validity and reliability compared to existing devices.

However, since the experiment was not applied to all age groups and patients with diseases, it was difficult to apply the results of the study to everyone. If a follow-up study that complements this limitation will be conducted in the future, the Spirokit can show high utilization as a substitute for the PFT device and the RMS test device used in existing clinical trials.

\section{Conflict of Interest}

The authors declared no potential conflicts of interest with respect to the authorship and/or publication of this article.

\section{References}

1. Rotar AM, van den Berg MJ, Kringos DS, Klazinga NS. Reporting and use of the OECD Health Care Quality Indicators at national and regional level in 15 countries. Int J Qual Health Care 2016;28:398-404.

2. Lopez AD, Shibuya K, Rao C, Mathers CD, Hansell AL, Held LS, et al. Chronic obstructive pulmonary disease: current burden and future projections. Eur Respir J 2006;27:397-412.

3. Jung EK. Chronic disease status and issues. Cheongju: Korea Centers for Disease Control and Prevention; 2019.

4. Salvi SS, Barnes PJ. Chronic obstructive pulmonary disease in non-smokers. Lancet 2009;374:733-43.

5. World Health Organization. Asthma [Internet]. Geneva: World Health Organization, 2020 [cited 2020 Jun 2]. Available from: https://www.who.int/news-room/q-a-detail/asthma.

6. Koh YJ, Kim SD, Park SY, Jang SK. Childrens' health risk assessment on indoor hazardous air pollutants of preschool facility. J Environ Health Sci 2009;35:78-85.

7. Haahtela T, Tuomisto LE, Pietinalho A, Klaukka T, Erhola M, Kaila M, et al. A 10 year asthma programme in Finland: major change for the better. Thorax 2006;61:663-70.

8. Gold WM, Koth LL. Pulmonary function testing. In: Broaddus VC, Mason RJ, Ernst JD, King TE, Lazarus SC, Murray JF, et al., editors. Murray and Nadel's textbook of respiratory medicine. 6th ed. Philadelphia (PA): W.B. Saunders; 2016. p. 407-435.e18.

9. Graham BL, Steenbruggen I, Miller MR, Barjaktarevic IZ, Cooper BG, Hall GL, et al. Standardization of spirometry 2019 update. An official American Thoracic Society and European Respiratory Society technical statement. Am J Respir Crit Care Med 2019;200:e70-88.

10. Hanayama K, Ishikawa Y, Bach JR. Amyotrophic lateral sclerosis. Successful treatment of mucous plugging by mechanical insufflation-exsufflation. Am J Phys Med Rehabil 1997;76:338-9.

11. Teixeira-Salmela LF, Olney SJ, Nadeau S, Brouwer B. Muscle strengthening and physical conditioning to reduce impairment and disability in chronic stroke survivors. Arch Phys Med Rehabil 1999;80:1211-8.

12. Teixeira-Salmela LF, Parreira VF, Britto RR, Brant TC, Inácio EP, Alcântara TO, et al. Respiratory pressures and thoracoabdominal motion in community-dwelling chronic stroke survivors. Arch Phys Med Rehabil 2005;86:1974-8.

13. Decramer M, Demedts M, Rochette F, Billiet L. Maximal transrespiratory pressures in obstructive lung disease. Bull Eur Physiopathol Respir 1980;16:479-90.

14. Agarwal V, Ramachandran N. Design and development of a low-cost spirometer with an embedded web server. Int J Biomed Eng Technol 2008;1:439-52.

15. Ovechkin A, Vitaz T, de Paleville DT, Aslan S, McKay W. Evaluation of respiratory muscle activation in individuals with chronic spinal cord injury. Respir Physiol Neurobiol 2010;173:171-8.

16. Ministry of SMEs and Startups. Bio health technology roadmap for SME 2020-2022 [Internet]. Daejeon: Ministry of SMEs and Startups, 2020 [cited 2020 Jun 2]. Available from: http://smroadmap. smtech.go.kr/0201/view/m_code/A140/s_code/A17/idx/2505. 
17. Shmulewitz A, Langer R, Patton J. Convergence in biomedical technology. Nat Biotechnol 2006;24:277.

18. Oh YM, Hong SB, Shim TS, Lim CM, Koh Y, Kim WS, et al. Effect of a new spirometric reference equation on the interpretation of spirometric patterns and disease severity. Tuberc Respir Dis 2006;60:215-20.

19. Balasubramaniam M, Sivapalan K, Thuvarathipan R. Lung function parameters of healthy Sri Lankan Tamil young adults. Ceylon Med J 2014;59:49-53.

20. Melbye H, Medbø A, Crockett A. The FEV1/FEV6 ratio is a good substitute for the FEV1/FVC ratio in the elderly. Prim Care Respir J 2006; 15:294-8.

21. Dassios T, Katelari A, Doudounakis S, Dimitriou G. Comparison of two methods of measurement of maximal respiratory pressures in health and cystic fibrosis. J Biomed Sci Eng 2013;6: 43-8.

22. Badr C, Elkins MR, Ellis ER. The effect of body position on max- imal expiratory pressure and flow. Aust J Physiother 2002;48: 95-102.

23. Portney LG. Foundations of clinical research: applications to evidence-based practice. 4th ed. Philadelphia (PA): FA Davis; 2020.

24. Bland JM, Altman DG. Statistical methods for assessing agreement between two methods of clinical measurement. Int J Nurs Stud 2010;47:931-6.

25. Jacobson NS, Truax P. Clinical significance: a statistical approach to defining meaningful change in psychotherapy research. J Consult Clin Psychol 1991;59:12-9.

26. COSMED. MicroQuark brochure [Internet]. Rome: COSMED, 2020 [cited 2020 Jun 2]. Available from: https://www.cosmed. com/hires/microQuark_flyerbrochure_A4_C09061-02-93_ EN_web.pdf.

27. CareFusion. The manual of MicroRPM. Basingstoke: CareFusion; 2014. 